Classification

Physics Abstracts

$42.55 \mathrm{Gp}-68.55-81.15 \mathrm{Fg}$

\title{
Microstructural Characterization of Thin Films Obtained by Laser Irradiation
}

\author{
Paolo Mengucci $\left({ }^{1}\right)$, Gianni Barucca $\left({ }^{1}\right)$, Renato Marzocchini $\left({ }^{1}\right)$ and Gilberto Leggieri $\left({ }^{2}\right)$ \\ $\left({ }^{1}\right)$ Dipartimento di Scienze dei Materiali e della Terra, Università di Ancona, Via Brecce Bianche, \\ 60131 Ancona, Italy \\ $\left(^{2}\right)$ Dipartimento di Fisica, Università di Lecce, Via Arnesano, 73100 Lecce, Italy
}

(Received March 1; accepted May 31, 1995)

\begin{abstract}
Thin films obtained by direct pulsed excimer laser irradiation and by laser reactive ablation were characterized by scanning electron microscopy, cross sectional transmission electron microscopy and grazing angle $\mathrm{X}$-ray diffraction. The results obtained were interpreted in function of the deposition parameters such as substrate temperature, pressure of the ambient atmosphere, number of laser pulses and laser fluence.
\end{abstract}

\section{Introduction}

Lasers are widely used for promoting synthesis and growth of thin films onto a specified site because of the many advantages of the laser based techniques with respect to the traditional ones. In particular, the accurate spatial control, the relative low deposition temperature, the high efficiency of the deposition process and the possibility of synthesizing multilayer structures with a single irradiation process are the most important advantages of the deposition techniques based on laser irradiation. Laser chemical vapour deposition, synthesis of compound under direct laser irradiation and congruent ablation deposition of compounds such as high critical temperature superconductors and titanium nitride are recent and successful methods for thin film formation $[1,2]$.

Although lasers have been used in materials processing for a long time, their potentialities in this field have not been fully exploited and new laser-based techniques are continuously improved. A very new laser-based technique named laser reactive ablation (LRA) is now emerging for the deposition of thin films. In the LRA deposition method a target, placed in a suitable atmosphere, is irradiated by a laser beam. This laser beam causes ablation of the target material and promotes chemical reactions between the ablated material and the atmosphere. The substrate which is placed at a certain distance from the target collects this ablated and reacted material $[3,4]$. 
The purpose of the present work is the study of microstructural characteristics of two different layers obtained by two different laser based techniques for the investigation of mechanisms responsible of the deposition processes. In particular, the results of the structural characterization of a multilayer titanium nitride/titanium silicide $\left(\mathrm{Ti}_{2} \mathrm{~N} / \mathrm{TiSi}_{2}\right)$ structure synthesized on silicon by laser direct synthesis (LDS) in a single step procedure and of a titanium carbide (TiC) layer deposited on silicon by LRA are reported.

Titanium nitrides result to be the best candidates for performance diffusion barriers to be used in very large scale integration (VLSI) technology since they combine the advantage of excellent electrical properties with extreme chemical inertness and high mechanical stability [5]. However the direct application of titanium nitride layers on bulk Si poses the risk of formation of a Schottky contact. It is possible to overcome this difficulty by interposing a layer of $\mathrm{TiSi}_{2}$ between the titanium nitride diffusion barrier and the Si substrate. The realization of multilayer structures of this type requires several subsequent operations consisting of deposition, masking and high temperature $\left(600 \div 900^{\circ} \mathrm{C}\right)$ thermal treatments for the formation of compounds. A different approach to silicide and nitride formation is to provide for the compound growth directly in the place of interest as a result of a laser-promoted reaction between the silicon bulk and a Ti film and between the Ti film and an ambient atmosphere containing nitrogen in a single step process [6].

Titanium carbide (TiC) films are used in many applications because of their high hardness and their high oxidation and wear resistance. The conventional deposition techniques most frequently used for the formation of TiC films are the hot wall chemical vapour deposition (CVD) and the laser-assisted CVD (LCVD). The main drawbacks of both techniques are the high temperature $\left(750 \div 800^{\circ} \mathrm{C}\right)$ necessary for the formation of the compound and the multi-step procedures. On the other side, the LRA deposition technique constitutes a different approach to the formation of TiC films because relatively low temperatures $\left(200 \div 300^{\circ} \mathrm{C}\right)$ are used for promoting the adhesion of the film to the substrate. Furthermore, this technique allows to deposit films by a single-step procedure [7].

\section{Experimental}

Irradiations were performed by a Lambda Physik LPX $315 \mathrm{i} \mathrm{XeCl} \mathrm{excimer} \mathrm{laser}(\lambda=308 \mathrm{~nm}$, $\tau_{\mathrm{FWHM}}=30 \mathrm{~ns}$ ) operating at different fluences and repetition rates. After deposition, samples were characterized by scanning electron microscopy (SEM), grazing angle X-ray diffraction (GAD) and cross sectional transmission electron microscopy (TEM) in order to investigate the dependence of film properties such as stoichiometry and thickness on the deposition parameters.

TEM observations were executed both on planar and cross sectional specimens. Samples were prepared by conventional techniques and the final thinning were carried out in ion beam by using Ar ions at $6 \mathrm{kV}, 0.5 \mathrm{~mA}$ and an incidence angle of $13^{\circ}$. GAD investigations were accomplished with a diffractometer in the asymmetric Bragg configuration at two different angles of incidence $\left(0.2^{\circ}\right.$ and $0.7^{\circ}$ ) of the $\mathrm{X}$-ray beam with respect to the sample surface for detecting possible differences in the depth composition of the deposited layers.

2.1 $\mathrm{Ti}_{2} \mathrm{~N} / \mathrm{TiSi}_{2}$ Multilayer. - Samples consisted of thin films of titanium $140 \mathrm{~nm}$ thick evaporated on a (100) Si single crystal wafer. Irradiations were performed in a chamber filled with $\mathrm{N}_{2}$ at a slightly superatmospheric pressure $(1100 \mathrm{mbar})$ to avoid contamination from the surrounding air. Nitrogen with a purity better than $99.998 \%$ and an oxygen content lower than 2 ppm were used. During irradiations the laser was operating at a fluence of $0.6 \mathrm{~J} / \mathrm{cm}^{2}$ and at repetition rate of $50 \mathrm{~Hz}$. Sequences of 100 to 5000 consecutive laser pulses were directed at the same location on the sample surface. 
2.2 TiC. - A pure $\mathrm{Ti}(99.6 \%)$ target was used for the LRA deposition of TiC films. Irradiations were performed with the laser operating at a fluence of $5 \mathrm{~J} / \mathrm{cm}^{2}$ and a repetition rate of $8 \mathrm{~Hz}$. Series of $10^{4}$ pulses were directed on the Ti target. During irradiations the target was rotating at a frequency of $3 \mathrm{~Hz}$. A Si (100) substrate placed at a distance $d=12.5 \mathrm{~mm}$ from the target collected the ablated material. The substrate was heated at $200{ }^{\circ} \mathrm{C}$ for increasing the adhesion of the deposited layer. Experiments were performed in methane $\left(\mathrm{CH}_{4}\right)$ at different values of the ambient pressure in the range $8 \times 10^{-6} \div 10$ mbar in order to investigate the influence of the deposition parameters on the film properties.

\section{Results}

3.1 $\mathrm{Ti}_{2} \mathrm{~N} / \mathrm{TiSi}_{2}$ MULTILAYER. - SEM observations allowed study the surface evolution of the samples as a function of the number of laser pulses. Results obtained for the samples submitted to 1000 and 5000 laser pulses, respectively, are shown in Figure 1. The sample submitted to 1000
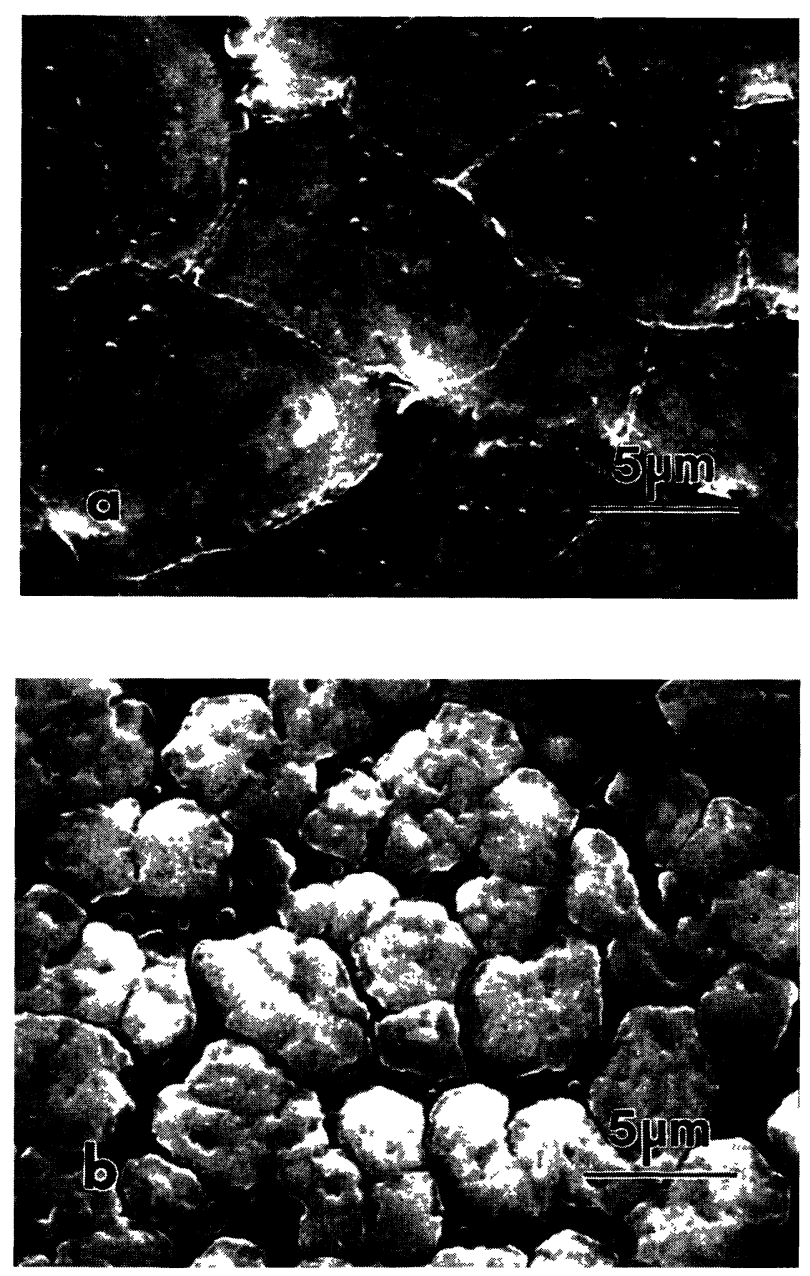

Fig. 1. - SEM micrographs of samples submitted to: a) 1000 laser pulses, b) 5000 laser pulses. 


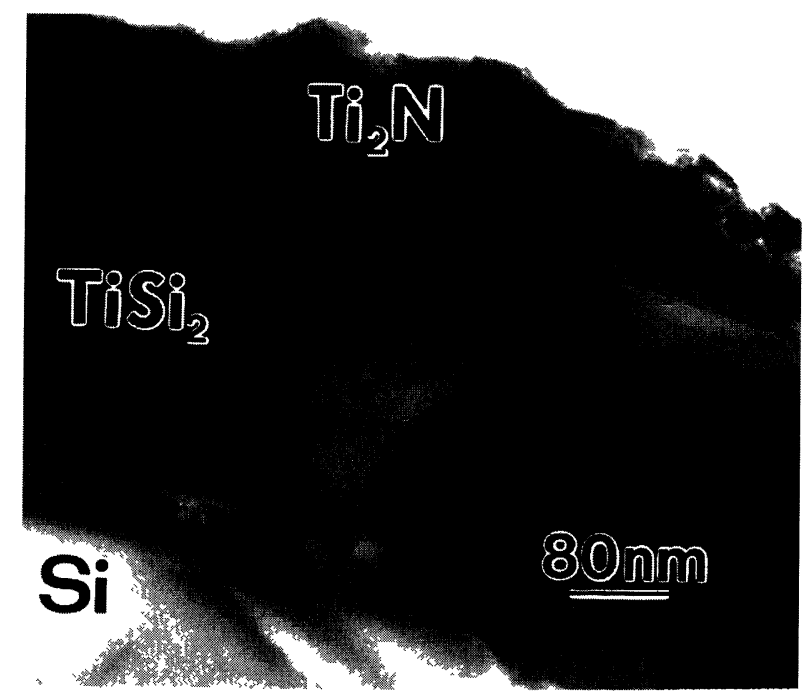

Fig. 2. - Cross sectional TEM image showing the deposited layers. Dislocations at the interface between the $\mathrm{TiSi}_{2}$ layer and the $\mathrm{Si}$ substrate are clearly visible.

laser pulses exhibits a surface consisting of an incipient grained structure, Figure 1a. Increasing the number of laser pulses causes the grains to become more and more defined. After 5000 pulses the surface consists of well resolved and separated grains with mean lateral dimensions of $3 \mu \mathrm{m}$, as shown in Figure 1b.

TEM cross section observations clearly evidenced the grained structure of the reacted layer. The grains appear well separated and their mean height is $0.3 \mu \mathrm{m}$. The inner structure of a grain is shown in Figure 2 where two layers showing different contrast are clearly visible. Selected area diffraction (SADP) and microdiffraction techniques allowed determination of structure and composition of the two layers. The surface layer is constituted of the $\mathrm{Ti}_{2} \mathrm{~N}$ phase having a tetragonal structure, space group I4 $1 /$ amd and lattice parameters $a=0.414 \mathrm{~nm}$ and $c=0.8805 \mathrm{~nm}$. Microdiffraction investigations carried out under different orientations showed that the second layer is constituted of the $\mathrm{TiSi}_{2}$ phase having an orthorhombic structure, space group $\mathrm{Cmcm}$ and lattice parameters $a=0.362 \mathrm{~nm}, b=1.376 \mathrm{~nm}$ and $c=0.3605 \mathrm{~nm}$. The surface layer $\left(\mathrm{Ti}_{2} \mathrm{~N}\right)$, made up of a very fine dispersion of microcrystals of about $50 \mathrm{~nm}$, shows a non uniform thickness extending from 50 to $150 \mathrm{~nm}$. The second layer $\left(\mathrm{TiSi}_{2}\right)$ has also a non uniform thickness and consists of crystals of about $250 \mathrm{~nm}$. The interface between the $\mathrm{Ti}_{2} \mathrm{~N}$ and the $\mathrm{TiSi}_{2}$ layers appears rather sharp and lacking in defects. On the contrary, the interface between the TiSi 2 and the Si bulk is broad and presents many defects such as dislocations and small segregated phases.

3.2 TiC. - SEM observations showed that samples ablated at pressure $p<10^{-5}$ mbar present high density of surface droplets with dimensions ranging from 0.1 to $15 \mu \mathrm{m}$. The largest droplets are always visible as aggregates of few members. An example is reported in Figure $3 a$ where the surface of the sample ablated at a pressure $p=8 \times 10^{-6} \mathrm{mbar}$ is shown. At the increasing of the $\mathrm{CH}_{4}$ pressure the mean dimension and the density of the surface droplets decrease as can be observed in Figure $3 \mathrm{~b}$ for the sample ablated at a pressure $p=2 \times 10^{-5}$ mbar.

$\mathrm{X}$-ray diffraction showed that samples ablated at ambient pressure $p<10^{-5}$ mbar mainly consists of hexagonal $\alpha$-Ti with lattice parameters $a=0.295 \mathrm{~nm}$ and $c=0.4686 \mathrm{~nm}$. Small amounts of 

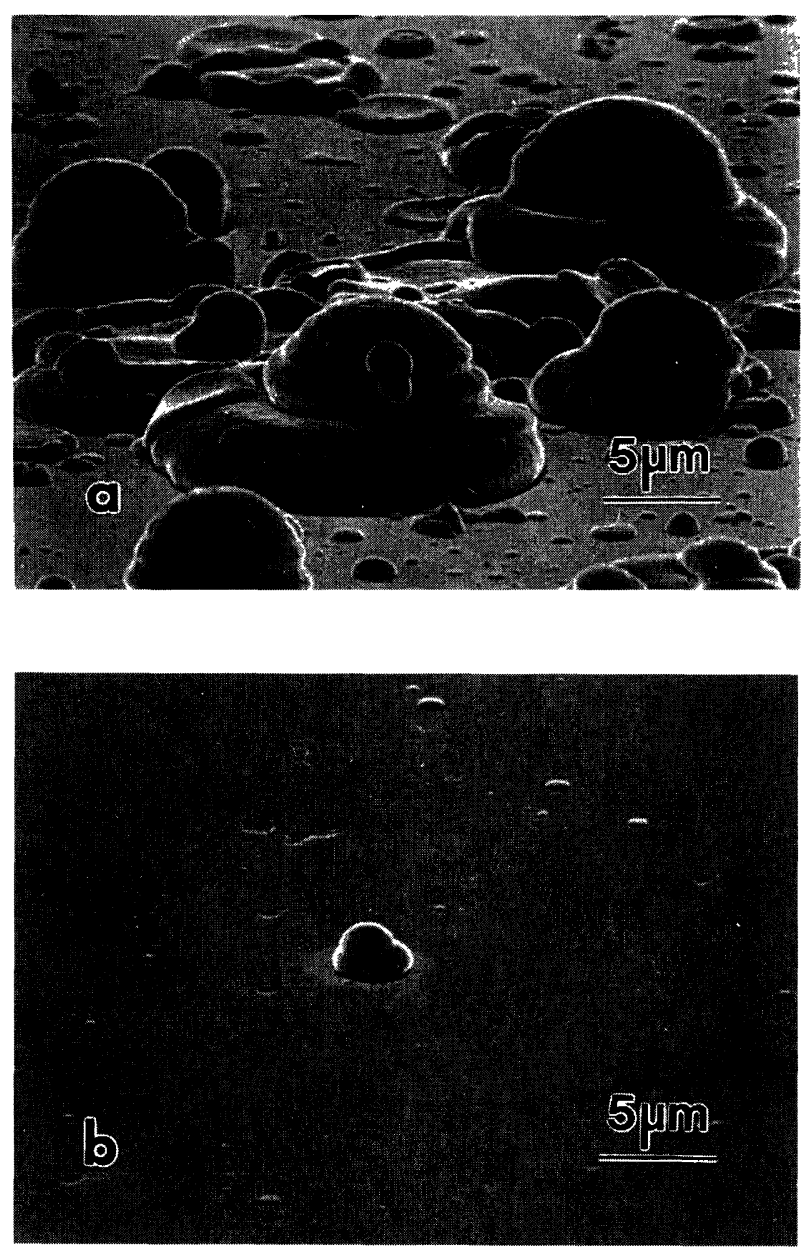

Fig. 3. - SEM micrographs of samples ablated in $\mathrm{CH}_{4}$ atmosphere at: a) $p=8 \times 10^{-6}$ mbar, b) $p=2 \times 10^{-5}$ mbar.

non-stoichiometric $\mathrm{TiC}$ were also found inside the deposited layers. Titanium oxides are present both near the surface and well inside the layer as can be observed in Figure $4 \mathrm{a}$ where the GAD spectra of the sample ablated at $p=8 \times 10^{-6}$ mbar are reported for two angles of incidence of the X-ray beam. Samples ablated at higher pressure exhibit a better stoichiometry of the TiC compound and a lower content of metallic Ti. Analyses showed that the most stoichiometric TiC (fcc with $a=0.43274 \mathrm{~nm}$ ) was obtained for the sample ablated at $p=2 \times 10^{-5}$ mbar. Figure $4 \mathrm{~b}$ reports the GAD spectra of this latter sample where the $\mathrm{TiC}$ peaks are indicated. Calculation of the crystallites size performed using the Scherrer formula applied to the $\mathrm{TiC}$ (200) peak gave $L_{200}=19.8 \mathrm{~nm}$. For pressure $p \geq 10^{-1}$ mbar samples show higher amounts of titanium oxides both near the surface and well inside the deposited layer whereas metallic $\mathrm{Ti}$ and $\mathrm{TiC}$ are completely absent.

Cross sectional TEM investigations showed that samples are constituted of a continuous layer formed of columnar grains originating at the interface with the substrate. The structure of the deposited layer relative to the sample exhibiting the best stoichiometry of the $\mathrm{TiC}$ compound is 

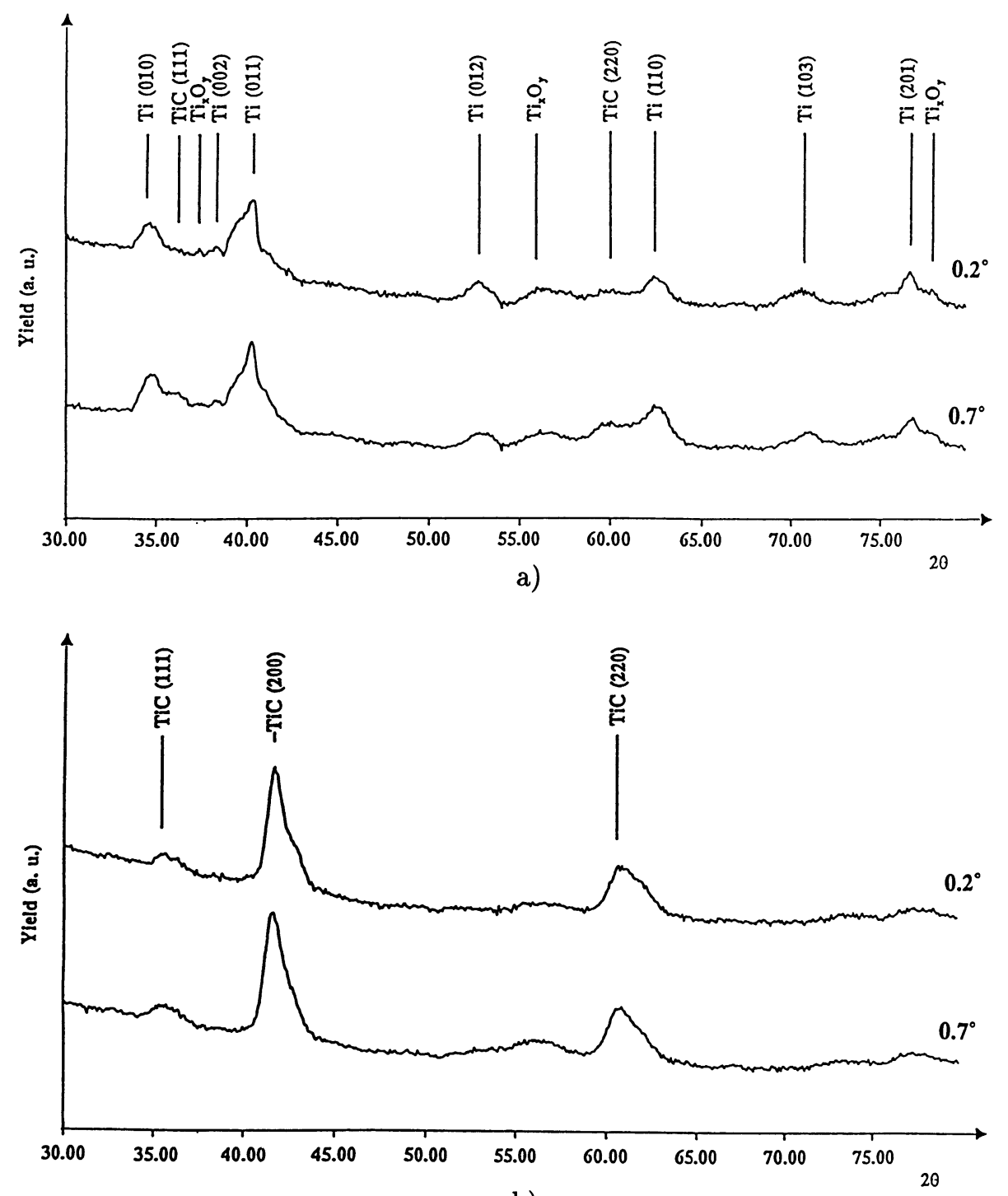

b)

Fig. 4. - X-ray diffraction spectra of samples ablated in $\mathrm{CH}_{4}$ atmosphere at: a) $p=8 \times 10^{-6}$ mbar, b) $p=2 \times 10^{-5}$ mbar. Spectra were taken at $0.2^{\circ}$ and $0.7^{\circ}$ of incidence of the X-ray beam with respect to the sample surface.

reported in Figure 5. In this case the continuous layer has a mean thickness of $80 \mathrm{~nm}$ and is formed of columnar grains having a mean lateral dimension of $20 \mathrm{~nm}$. Rutherford backscattering spectroscopy (RBS) analyses performed on samples deposited at different ambient partial pressure showed that at the increasing of the partial pressure the thickness of the deposited layer decreases. 


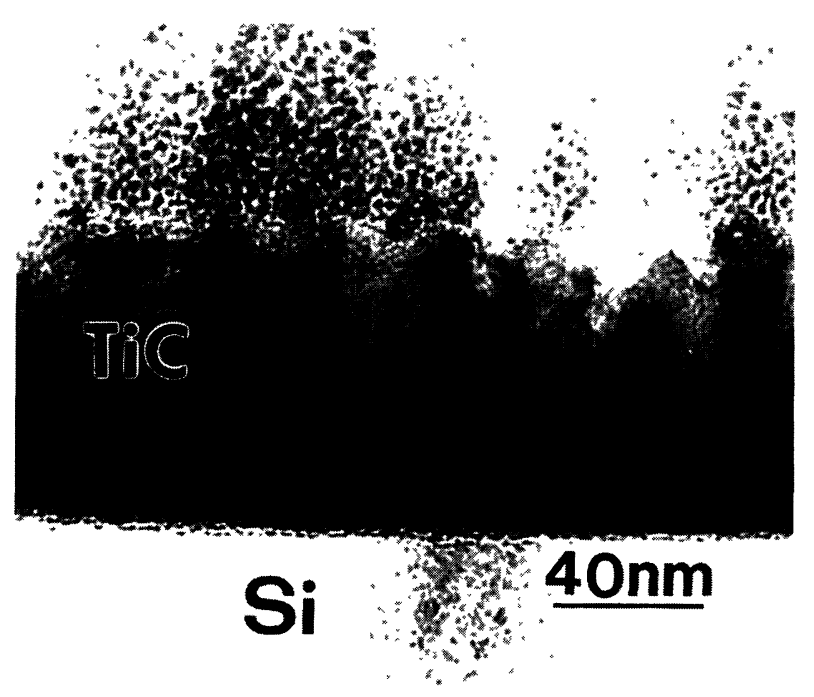

Fig. 5. - Cross sectional TEM image of the layer exhibiting the best stoichiometry of the TiC compound ablated at a pressure $p=2 \times 10^{-5}$ mbar of the $\mathrm{CH}_{4}$ atmosphere.

\section{Discussion}

4.1 $\mathrm{Ti}_{2} \mathrm{~N} / \mathrm{TiSi}_{2}$ Multilayer. - Results put into evidence that a multilayer structure consisting of a titanium nitride layer superimposed to a titanium silicide one was obtained by a single step LDS procedure. Furthermore, the absence of both titanium and silicon oxides and of material expelled from the irradiated zone should be mentioned.

SEM results pointed out that surface perturbation increases with the number of laser pulses. This evolution of the surface morphology towards a rougher structure at the increasing of the number of laser pulses suggest that the reactions leading to the formation of the superimposed layers take place in a liquid phase. Numerical computations of the sample surface temperature, performed by solving the heat diffusion equation, confirmed these results and showed that the laser irradiation causes melting of the entire Ti film and of a layer of the Si substrate. In this way the titanium nitride formation takes place in a liquid phase at the surface of the layer as a result of a chemical reaction between the Ti layer and the ambient atmosphere. On the other side, the silicide forms for reaction between the $\mathrm{Ti}$ and the melted $\mathrm{Si}$ layer [8]. These considerations allow to justify the presence of dislocations at the $\mathrm{TiSi}_{2} / \mathrm{Si}$-bulk interface, as evidenced by TEM observations, since during solidification the $\mathrm{TiSi}_{2} / \mathrm{Si}$-bulk interface separates a liquid phase $\left(\mathrm{TiSi}_{2}\right)$ and a solid one (Si-bulk). On the contrary, the $\mathrm{Ti}_{2} \mathrm{~N} / \mathrm{TiSi}_{2}$ interface that, during solidification, separates two liquid phases results to be dislocation free. The presence of these dislocations at the interface between the reacted layer and the Si substrate could be detrimental for the electrical performances of the multilayer.

4.2 TiC. - Results obtained from GAD and electron microscopy investigations showed that the surface morphology of the layer and the stoichiometry of the compound are strongly dependent on the ambient partial pressure during deposition. Furthermore, the presence of droplets on the surface of samples suggest that the carbidation process could occur in a liquid phase. This is further confirmed by numerical simulations of the target thermal behaviour under multipulse laser irradiation which indicate that the target surface melts during each laser pulse. Calculations 
also showed that the carbidation process cannot occur by pure heterogeneous reaction between the melt and the ambient atmosphere since its low reaction rate is unable to justify the thickness of the obtained TiC layers. Pure hydrodynamic effects responsible for fast mixing of the melted layer resulting in an efficient carbidation process are to be considered $[9,10]$. It is obvious that the efficiency of the carbidation process is linked to the quantity of carbon-containing gas provided on the target surface. This latter observation justify the dependence of the TiC layers stoichiometry on the partial pressure of the $\mathrm{CH}_{4}$ atmosphere as observed in our experimental results.

Gas phase reaction of the evaporated titanium with the carbon-containing atmosphere must be also considered. In particular, chemical reactions could occur within the vapour plume as a consequence of the vibrational excitations of the $\mathrm{CH}_{4}$ molecules by plasma electrons. So both liquid phase and gas phase reactions could contribute to the synthesis of TiC.

Turbulence of the ambient atmosphere and scattering phenomena reduces the growth rate of the TiC layer. So, at the increasing of the ambient pressure the thickness of the deposited layers decreases, as was observed in our experiments.

The presence of large surface droplets suggests that the most important mechanism in the carbidation process is the liquid phase reaction that takes place on the target surface. The gas phase reaction, even if not negligible, gives a limited contribution to the synthesis of TiC molecules.

\section{Conclusions}

The microstructural characteristics of a multilayer titanium nitride/titanium silicide $\left(\mathrm{Ti}_{2} \mathrm{~N} / \mathrm{TiSi}_{2}\right)$ structure synthesized on silicon by laser direct synthesis (LDS) in a single step procedure and of a titanium carbide (TiC) layer deposited on silicon by LRA were investigated by different techniques. Microstructural observations, integrated by numerical computation of the sample or target thermal behaviour, allowed to obtain information on the possible processes responsible for the layer formation. Results were interpreted considering the following:

- in the LDS deposition method the reaction takes place in a liquid phase at the sample surface and the structure of the deposited layers were interpreted in function of the deposition parameters;

- in the LRA deposition method the compound forms on the target surface in a liquid phase whereas the gas phase reaction gives a limited contribution.

\section{References}

[1] Boyd I.W., Laser Processing of Thin Films and Microstructure (Springer, Berlin, 1987).

[2] D’Anna E., Leggieri G. and Luches A., Thin Solid Films 218 (1992) 219.

[3] Fogarassy E., Slaoui A., Fuchs C. and Stoquert J.P., Appl. Surf. Sci. 46 (1990) 195.

[4] Vega F., Afonso C.N., Ortega C. and Siejka J., J. Appl. Phys. 74 (1993) 963.

[5] Von Almen M. and Lau S.S., Laser Annealing of Semiconductors, J.M. Poate and J.W. Mayer Eds. (Academic Press, New York, 1982).

[6] Mihailescu I.N., Chitica N., Lita A., Teodorescu V.S., Luches A., Leggieri G., Martino M., Majni G. and Mengucci P., Thin Solid Films 251 (1994) 23.

[7] D’Anna E., De Giorgi M.L., Leggieri G., Luches A., Majni G., Martino M., Mihailescu I.N. and Mengucci P., J. Phys. IV France 4 (1994) C4-51.

[8] D'Anna E., Leggieri G. and Luches A., Appl. Phys. A 45 (1988) 325.

[9] Arutyunan R.V., Action of Laser Radiation on Materials (Nauka, Moscow, 1989).

[10] Dorofeev A. and Libenson M.N., Sov. J. Chem. Phys. 11 (1992) 4. 